\title{
LA GESTION ASSOCIATIVE DE LA PÊCHE EN FRANCE : UNE ANALYSE ÉCONOMIQUE À PARTIR DU CAS DES SALMONIDÉS
}

\author{
P. LE GOFFE, J. SALANIÉ
}

\begin{abstract}
Agrocampus Rennes, Département d'Économie Rurale et Gestion, 65, rue de Saint-Brieuc, CS 84215, 35042 Rennes cedex. Tél. : 02234854 16 - Fax : 0223485417 Courriel : legoffe@agrocampus-rennes.fr
\end{abstract}

Reçu le 28 juin 2004

Accepté le 24 janvier 2005

Received June 28, 2004

Accepted January 24, 2005

\section{RÉSUMÉ}

L'analyse économique et l'examen des quelques données empiriques disponibles en France incitent à se demander si la gestion associative permet de tirer suffisamment de bien-être de la pêche de loisir en eau douce. Notre analyse s'appuie sur le cas des salmonidés. D'une part, l'effort de pêche est probablement trop important sur les bons parcours faciles d'accès, ce qui peut entraîner des pertes de bien-être dues à la congestion. D'autre part, les propriétaires riverains ne sont pas suffisamment incités à améliorer la qualité de l'environnement, alors que les pêcheurs ont un consentement à payer pour des améliorations. Ces inefficacités s'expliquent par la conjonction de trois phénomènes. Tout d'abord, les droits de propriété, privés, sont atténués par des dispositions législatives. Ensuite, les associations ne contrôlent pas suffisamment l'effort de pêche individuel et collectif. Finalement, les politiques halieutiques sont centrées sur la protection de la ressource et sont, bien que nécessaires, insuffisantes pour maximiser la rente sociale tirée de la pêche. La régulation de l'effort de pêche et la négociation de certains services environnementaux sur la base du principe bénéficiaire-payeur permettraient d'améliorer le bien-être collectif. Des tarifs sociaux permettraient de maintenir l'équité dans l'accès.

Mots-clés : accès libre, congestion, coûts de transaction, environnement, pêche récréative, droits de propriété, bénéficiaire-payeur, institutions.

\section{RECREATIONAL FISHING MANAGEMENT IN FRANCE: AN ECONOMIC ANALYSIS WITH EMPHASIS ON SALMONIDS}

\begin{abstract}
On behalf of economic analysis and some French empirical data, it is questionable that the associative management of freshwater recreational fishing in France is leading to collective welfare maximization. Our analysis relies on the salmonids case. On one hand, fishing effort is probably too high at the best and easy to access fishing sites, which can lead to welfare losses due to overcrowding. On the other, riverside landowners are not sufficiently incited to improve environmental quality while anglers express a positive willingness to pay for improvements. These inefficiencies are explained by the conjunction of three phenomena. First, private property rights are attenuated under the pressure of legislative dispositions. Second, the angling associations do not control enough individual and collective fishing effort. Finally, halieutic policies focus on resource protection and are, while necessary, not enough to maximize the social rent associated with recreational
\end{abstract}


fishing. The regulation of fishing effort and the bargain of some environmental goods and services on the basis of beneficiary-pays principle could help to improve collective welfare. Equity in access could be insured through social pricing.

Key-words: open access, congestion, transaction costs, environment, recreational fishing, property rights, beneficiary-pays principle, institutions.

\section{INTRODUCTION}

A une époque où la vie, le temps et la nature ont une valeur croissante, les activités récréatives liées aux ressources naturelles sont généralement en plein essor. A l'inverse, la pêche en eau douce, activité récréative traditionnelle, semble connaître une certaine désaffection dans notre pays, si on se fie aux statistiques sur la pêche associative.

II n'est pas facile de faire un constat sur la pratique de la pêche récréative en France, car les données qui existent ici et là ne sont pas centralisées et régulièrement mises à jour. La dernière enquête nationale sur la pêche en eau douce date de 1991 (CSP, 1992). On y dénombrait cinq millions de pratiquants, dont trois millions de pêcheurs actifs pêchant plus de cinq jours par an. Ces chiffres sont supérieurs au 1,9 million de pêcheurs qui acquittaient la taxe piscicole en 1990, taxe nécessaire pour pêcher dans les eaux libres généralement gérées par les associations. La différence s'explique par les pêcheurs en eaux libres exonérés (15\% environ), et par ceux qui pratiquent dans les eaux closes non soumises à la réglementation, dont la gestion est privée. Cette enquête avait notamment pour objectif d'expliquer la baisse régulière du nombre de pêcheurs associatifs, qui s'est poursuivie depuis au rythme de 40000 pêcheurs en moins chaque année, provoquant la crise du financement du Conseil Supérieur de la Pêche ${ }^{1}$. A l'inverse, les effectifs de la pêche privée seraient en progression. L'avenir de la pêche associative fait actuellement l'objet d'une réflexion dans le cadre de la réforme de la loi sur l'eau.

La perte de 1,5 million de pêcheurs associatifs depuis 1964 serait d'abord due aux évolutions socio-économiques du pays (BRETON, 1993) : baisse de la population ouvrière, exode rural et vie de plus en plus urbaine, concurrence avec d'autres formes de loisirs et de vacances qui ont bénéficié d'une meilleure promotion. Les perturbations des milieux aquatiques sont également évoquées, sans qu'on puisse toujours les relier à l'évolution du nombre de pêcheurs. En matière de qualité de l'eau par exemple, on observe la diminution des points noirs du fait de la régression des pollutions industrielles et domestiques, mais aussi la disparition progressive des eaux les plus pures (IFEN, 1998). L'agriculture est mise en cause pour la modification des paysages et des écosystèmes, la progression des pollutions diffuses, des surfaces irriguées et de la déprise, cette dernière affectant l'entretien des berges et l'accès aux rivières.

Malgré un éphémère développement dans les années 60 , le tourisme pêche n'a pas réussi à enrayer le déclin des effectifs de pêcheurs en zone rurale. La Fédération française des guides de pêche dénonce la concurrence rude des parcours étrangers et la surexploitation des bons parcours nationaux susceptibles d'intéresser la clientèle nationale et internationale (AFIT, 1996). Ceci explique le déséquilibre des flux de touristes entre la France et l'étranger. Les touristes étrangers, qui recherchent des parcours associant gestion piscicole rigoureuse et densité faible de pêcheurs, sont peu nombreux en France (LAFAGE, 1992). En revanche, les pêcheurs français vont beaucoup à l'étranger. Une enquête a montré qu'ils étaient très intéressés par des parcours spécifiques de qualité, les plus naturels possibles, qui ne sont pas assez répandus dans notre pays (ANONYME, 1996).

\footnotetext{
${ }^{1}$ Etablissement public qui utilise le produit de la taxe piscicole dont il dispose pour la mise en valeur et la surveillance du domaine piscicole national (article L434-1 du code de l'environnement).
} 


\section{Tableau I}

Opinion des pêcheurs sur la congestion des rivières à saumon de l'Ouest.

\section{Table I}

Anglers opinion about congestion on salmon rivers in western France.

\begin{tabular}{|l|c|c|}
\hline \multicolumn{1}{|c|}{ Congestion } & $\begin{array}{c}\text { Effectifs } \\
\text { Number }\end{array}$ & $\%$ \\
\hline Très peu fréquenté / Not congested at all & 91 & 6,97 \\
Peu fréquenté / Congestion under average & 233 & 17,85 \\
Moyennement fréquenté / Acceptable congestion & 445 & 34,10 \\
Beaucoup fréquenté / Very congested & 375 & 28,74 \\
Trop fréquenté / Congestion is too high & 161 & 12,34 \\
\hline Total & $\mathbf{1 3 0 5}$ & $\mathbf{1 0 0 , 0 0}$ \\
\hline
\end{tabular}

Source : SALANIÉ, SURRY et LE GOFFE (2005).

On peut effectivement suspecter une exploitation trop importante des parcours associatifs facilement accessibles, car la pression de pêche n'est pas régulée. Cependant, il existe très peu de données sur la congestion des parcours de pêche en France. L'enquête nationale de 1990 montrait que $38 \%$ des pêcheurs et $58 \%$ de la sous-population des pêcheurs "sportifs" ne souhaitaient pas l'augmentation du nombre de pêcheurs. Les pêches nécessitant de l'espace, comme celle des salmonidés sont particulièrement sensibles à la congestion. C'est ainsi que des pêcheurs de saumons de l'Ouest, enquêtés par SALANIÉ, SURRY et LE GOFFE (2005) déplorent la fréquentation excessive de certaines rivières (Tableau I). En réponse à cette qualité insuffisante, on observe un mouvement de privatisation ${ }^{2}$ des parcours associatifs les plus côtés, concernant les espèces à forte demande comme le saumon ou la truite, un peu à la manière de ce qui s'est produit pour la chasse.

$\mathrm{Au}$ vu de ce constat, on peut se demander pourquoi notre pays ne tire pas davantage de richesse de la pratique de la pêche récréative, à l'instar de ce qui se passe dans les pays du nord de l'Europe. Cette question est intéressante si on veut répondre aux enjeux qui, au-delà de la pêche, concernent d'autres activités récréatives basées sur les ressources naturelles:

- amélioration du bien-être des pratiquants,

- développement des territoires ruraux et montagnards,

- multifonctionnalité de l'agriculture à l'heure où le soutien traditionnel des marchés est remis en question.

Alors que la pêche est un sujet empirique majeur pour les économistes de l'environnement anglo-saxons, on recense peu d'études françaises (BONNIEUX et VERMERSCH, 1993 ; DESAIGUES, LESGARDS et LISCIA, 1999 ; ARMAND, BONNIEUX et CHANGUEUX, 2002). Ces travaux précurseurs portent sur la mesure des bénéfices récréatifs et abordent assez peu les questions touchant à la régulation de l'activité ${ }^{3}$. II y a pourtant un intérêt scientifique à comprendre pourquoi la pêche récréative n'est pas mieux exploitée dans notre pays, alors qu'elle fait l'objet de droits de propriété parfaitement définis et de politiques de régulation anciennes.

\footnotetext{
${ }^{2}$ Le propriétaire révoque le bail qui le lie à une association et loue ses lots à des particuliers.

${ }^{3}$ Notons également les travaux de BRUN et PINET (2004) qui, avec une approche géographique, passent en revue les causes du déclin de la pêche ainsi que les réponses possibles.
} 
Le premier objectif de l'article est d'expliquer l'origine des inefficacités qui affectent la pêche récréative en France. Le deuxième objectif est d'introduire une réflexion sur les mesures qui permettraient de résorber certaines de ces inefficacités. Activité souvent non marchande, la pêche récréative nécessite la gestion simultanée du milieu et des habitats piscicoles, de la ressource halieutique et de l'effort de pêche. C'est pourquoi nous appuyons notre démonstration sur les théories économiques de l'environnement et des ressources naturelles, à l'intérieur du cadre analytique de l'économie publique. Il s'agit d'une analyse exploratoire qui, nous l'espérons, pourrait servir de point de départ à une discussion au sein des institutions de la pêche associative.

La première partie de l'article résume les travaux économiques sur la gestion optimale des activités récréatives et de la pêche en particulier. Dans la deuxième partie on confronte ce cadre théorique à l'organisation institutionnelle de la pêche et à la législation halieutique en France, en mettant l'accent sur la question des droits de propriété. La dernière partie s'intéresse aux instruments et dispositifs qui permettraient d'améliorer l'efficacité économique de la pêche de loisir, sans perdre l'équité dans l'accès que procure la gestion associative.

\section{MODÉLISATION ÉCONOMIQUE ET GESTION OPTIMALE DE LA PÊCHE DE LOISIR}

Pour les économistes, la gestion optimale d'un actif naturel fait référence à la notion d'optimum économique, qui correspond à la maximisation du bien-être collectif tiré de l'actif en question. La mesure monétaire du bien-être est fournie par le consentement à payer pour jouir de l'actif, que l'on obtient à partir de la fonction de demande des individus. S'agissant d'un actif à usage récréatif, comme un site naturel, la demande individuelle mesure la réaction de la fréquentation du site par un individu (nombre annuel de visites par exemple) à des variations du prix du droit d'entrée.

Dans le cas de la pêche, elle est obtenue empiriquement à partir d'un échantillon de pêcheurs fréquentant un site de pêche. On explique le nombre individuel de sorties par le coût de déplacement pour se rendre sur le site et d'autres caractéristiques individuelles comme le revenu, l'âge, la catégorie socioprofessionnelle, l'expérience récréative, la pratique d'activités et de sites de substitution (ainsi que leur coût d'accès). Par la suite, on considère que l'instauration d'un droit d'entrée aurait le même effet sur la fréquentation qu'une augmentation équivalente du coût de déplacement. Les économistes préfèrent raisonner sur la demande inverse, qui exprime le prix en fonction du nombre de sorties, car elle s'interprète comme le consentement à payer (CAP) marginal pour effectuer une sortie supplémentaire (Figure 1). II est élevé pour les premières sorties et de plus en plus faible au fur et à mesure que la fréquentation augmente. La figure 1 montre comment un pêcheur ajuste sa fréquentation $\mathrm{V}_{\mathrm{i}}$ à son coût de déplacement $\mathrm{C}_{\mathrm{i}}$. On peut alors définir la dépense annuelle du pêcheur pour aller à la pêche $\left(V_{i} C_{i}\right)$, ainsi que son CAP total qui correspond à la somme des CAP marginaux jusqu'à $V_{i}$, c'est à dire à l'aire sous la fonction de demande inverse jusqu'à $V_{i}$. L'excédent de CAP par rapport à la dépense s'interprète comme la mesure monétaire du bien-être du pêcheur, appelé surplus. La demande agrégée adressée à un site s'obtient en sommant les demandes individuelles.

On recense quelques travaux d'économie publique sur la gestion optimale des activités récréatives, comme ceux précurseurs de FISHER et KRUTILLA (1972) ou plus récents de SIBLY (2001). Dans la lignée de ces modèles, ANDERSON (1983 et 1993) a formalisé le cas de la pêche récréative. A la différence des modèles de pêcherie commerciale qui s'intéressent au comportement des producteurs, le modèle initial d'ANDERSON (1983), repose sur la spécification de la fonction de demande de visites agrégée adressée à un site de pêche. Les fonctions de demande inverse représentées à la figure 2 s'interprètent comme le consentement à payer marginal en plus des coûts de déplacement, c'est à dire la réaction à un droit d'entrée. En l'absence de droit d'entrée 
Coût de déplacement unitaire

CAP marginal

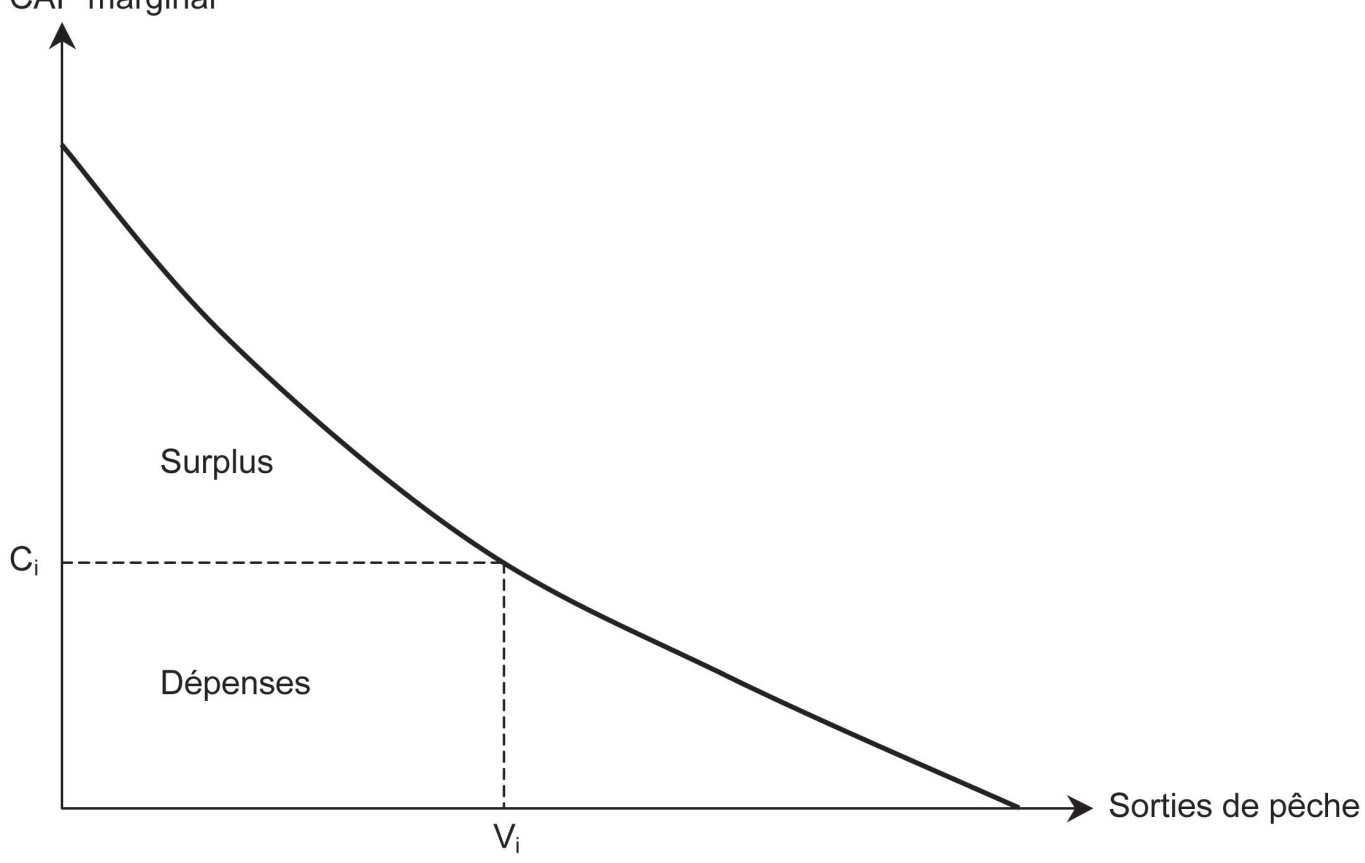

\section{Figure 1}

Demande individuelle inverse de sorties de pêche, dépenses et surplus.

\section{Figure 1}

Inverse individual demand for fishing days, spendings and surplus.

(prix nul), le nombre de visites agrégé est $\bar{D}$. Pour tenir compte du fait que les pêcheurs peuvent se nuire mutuellement (les économistes parlent d'externalités ${ }^{4}$ négatives), ANDERSON introduit la notion de demande à effort constant (courbes $\mathrm{KK}_{\mathrm{i}}$ de la figure 2). Au fur et à mesure que l'effort total augmente, les pêcheurs se situent sur des courbes de demande inverse inférieures, ce qui traduit la baisse de leur consentement à payer avec l'encombrement des parcours. C'est ainsi que $\mathrm{KK}_{2}$ est en dessous de $\mathrm{KK}_{1}$, car l'effort total $D_{2}$ constant sur $K K_{2}$ est plus grand que $D_{1}$. Si on appliquait un droit d'entrée, on n'observerait pas les courbes $\mathrm{KK}_{\mathrm{i}}$, qui sont pourtant les véritables fonctions de demande. Comme la courbe $\mathrm{KK}_{\mathrm{i}}$ change à chaque réduction de l'effort, on observerait seulement le lieu des points où l'effort total est confondu avec celui qui définit chaque courbe $\mathrm{KK}_{\mathrm{i}}$, c'est à dire la courbe $\mathrm{CC}$ de demande observée.

La demande inverse, correspondant à la courbe $\mathrm{KK}_{\mathrm{i}}$ peut s'écrire :

$$
P_{i}=P\left(D, X\left(D_{i}\right)\right)
$$

où $\mathrm{P}_{\mathrm{i}}$ est indifféremment le prix ou le consentement à payer marginal, $\mathrm{D}$ est le nombre total de visites et $X$ représente la qualité de la pêche, elle-même fonction de l'effort total $D_{i}$ qui est maintenu constant sur la courbe $K_{\mathrm{i}}$. La qualité de la pêche est fonction de paramètres qui dépendent de l'effort total, comme les captures par unité

\footnotetext{
${ }^{4}$ L'externalité est une interaction entre agents qui se produit hors du marché, c'est-à-dire sans transaction et compensation monétaire. Elle peut être négative comme ici, ou positive (cas des services environnementaux fournis gratuitement par les agriculteurs à la société).
} 
Prix d'un droit d'entrée,

CAP marginal

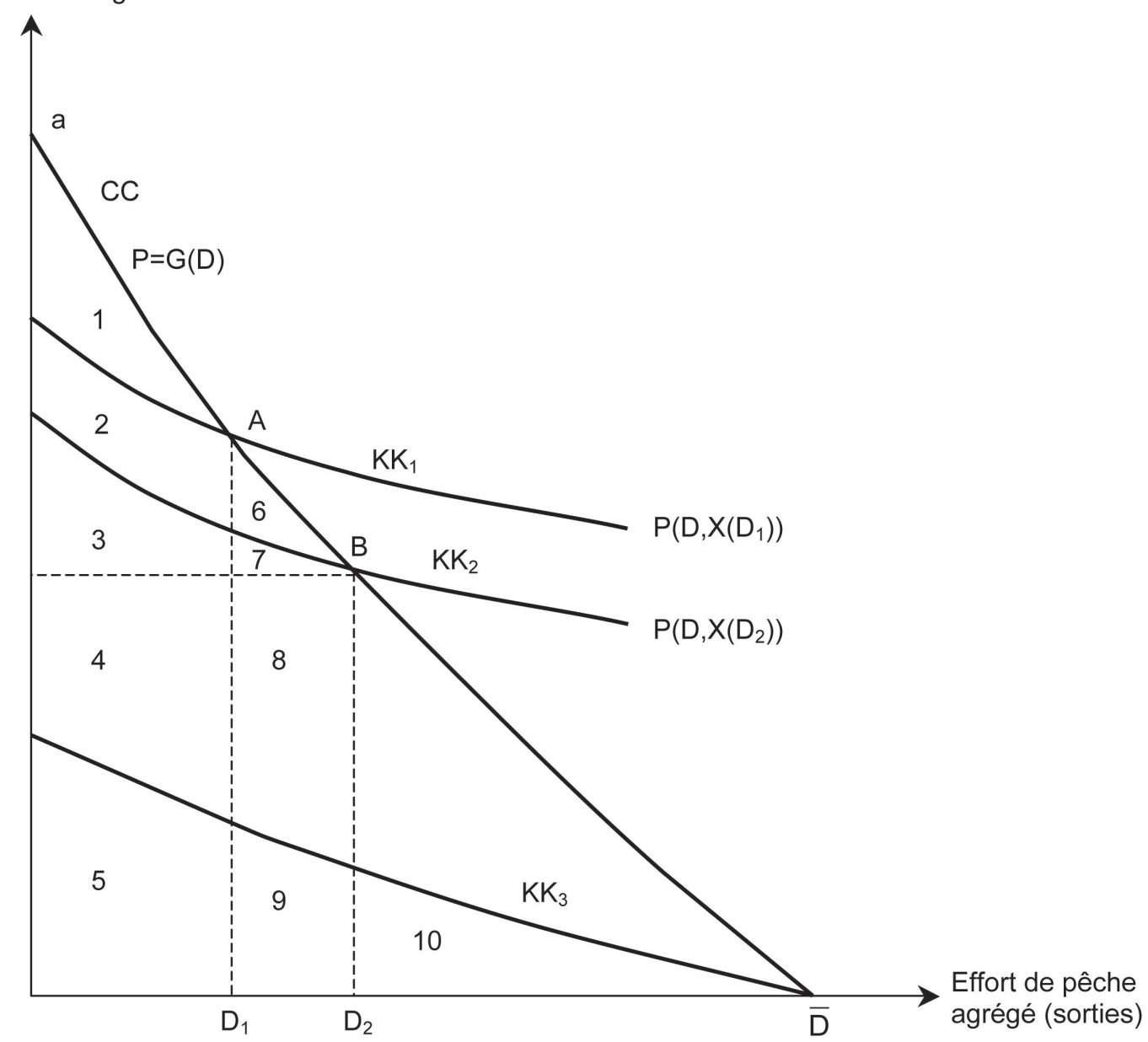

Figure 2

Fonctions de demande de pêche agrégées à externalité constante et équilibre de libre-accès.

\section{Figure 2}

\section{Constant externality aggregated fishing demand curves and open-access equilibrium.}

d'effort, la taille moyenne des captures et la tranquillité du parcours. Le lien entre la qualité et l'effort de pêche est supposé négatif, ce qui correspond à des externalités de stock ou de congestion qui affectent toutes deux la demande.

En l'absence de mesures de limitation de l'effort individuel, que nous qualifierons " d'accès libre 5 ", les pêcheurs continuent à visiter tant que les visites supplémentaires leur procurent du bien-être. L'effort total se fixe alors au niveau $\bar{D}$ qui annule le consentement à payer marginal pour la dernière journée de pêche (Figure 2), soit :

$$
P(\bar{D}, X(\bar{D}))=0
$$

\footnotetext{
${ }^{5}$ Dans la réalité associative française, il s'agit plutôt d'accès non contrôlé plutôt que d'accès libre, puisqu'il existe des périodes de fermeture, des limitations de taille et parfois des TAC. D'autre part, les pêcheurs acquittent un permis forfaitaire.
} 
Autrement dit, les pêcheurs arrêtent d'aller à la pêche quand ils ne sont plus prêts à payer pour une visite supplémentaire. Le bien-être collectif de la pêcherie est alors égal à la somme des CAP marginaux jusqu'à $\bar{D}$, soit l'aire sous la courbe $K K 3(5)+(9)+(10)$. On voit graphiquement que le bien-être collectif est plus élevé quand on limite l'effort total à $D_{2}$ ou même $D_{1}$ (intégrale des CAP marginaux jusqu'à $D_{2}$ et $D_{1}$ ). L'effort optimal, qui maximise le bien-être en ce qui concerne la pêcherie doit être déterminé à partir des courbes $\mathrm{KK}_{\mathrm{i}}$ de demande à effort constant. Si l'effort de pêche est $\mathrm{D}_{1}$, alors le surplus (ou bien-être) total en ce qui concerne la pêcherie correspond à l'aire délimitée par la courbe $K K_{1}$ et la verticale de $D_{1}$. Pour un effort $D_{1}$ le surplus est donc égal à (2) + (3) + (4) + (5). $\mathrm{Si}$ l'effort est augmenté jusqu'à $D_{2}$, le nouveau surplus équivaut à $(3)+(4)+(5)+(7)$ $+(8)+(9)$. Le surplus a diminué de (2), ce qui traduit la perte de bien-être associée à l'augmentation des externalités de stock et de congestion. En revanche, l'augmentation du nombre de visites de $D_{1}$ à $D_{2}$ génère un surplus additionnel équivalent à $(7)+(8)+(9)$. Graphiquement, on remarque aisément qu'à partir d'un certain point ( $D^{*}$ sur la figure 3$)$, la perte de surplus liée aux externalités entre pêcheurs est supérieure au gain de bienêtre tiré des visites supplémentaires. En conséquence, l'effort doit être amené jusqu'au niveau où le consentement à payer pour la dernière journée de pêche est égal à la perte de bien-être imposée à tous les pêcheurs du fait de cette augmentation marginale de l'effort. Comme l'externalité est négative, l'effort optimal est inférieur à l'effort " d'accès libre », qualifié de "tragédie des communs » par HARDIN (1968). La figure 3 représente la variation du surplus collectif, c'est-à-dire de l'aire sous les courbes $\mathrm{KK}_{\mathrm{i}}$, en fonction de l'effort total. On voit que l'effort total $D^{*}$ est inférieur à l'effort « d'accès libre » $\bar{D}$, pour lequel il subsiste malgré tout un surplus, à la différence de ce qui se passe pour les pêcheries commerciales.

Le modèle d'ANDERSON a-t-il été validé empiriquement ? Sur les multiples études économétriques anglo-saxonnes consacrées à la pêche récréative, on recense très peu de travaux sur la congestion, qui nécessitent de vastes enquêtes sur plusieurs sites. KERKVLIET et NOWELL (2000) et SCHUHMANN et SCHWABE (2004) trouvent un effet de la congestion sur la demande qui n'est pas purement négatif, mais parabolique. Le CAP et donc la satisfaction augmentent d'abord, puis diminuent au-delà d'un certain

Bien-être collectif de la pêcherie

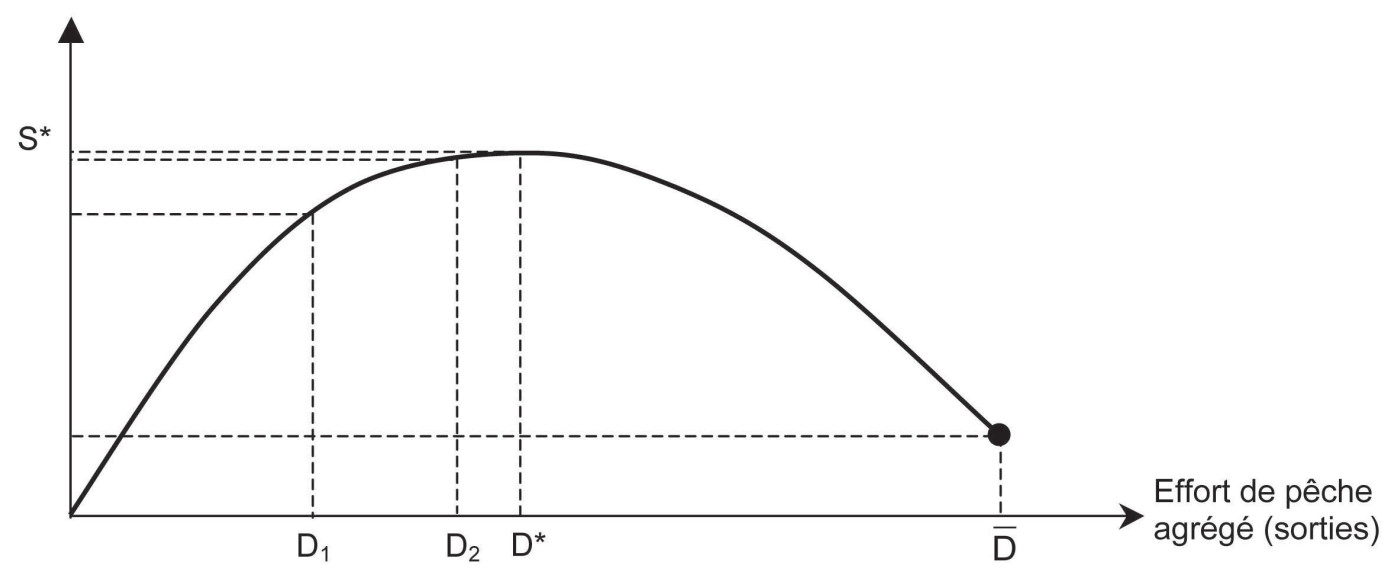

Figure 3

Bien-être collectif en fonction de l'effort de pêche total.

Figure 3

Collective welfare function of total fishing effort. 
effort collectif. En France, SALANIÉ, SURRY et LE GOFFE (2004) obtiennent des résultats similaires en estimant les fonctions de demande de pêche au saumon, à partir d'un échantillon de 828 pêcheurs pratiquant sur 28 rivières de l'ouest armoricain. On peut expliquer cet effet par l'existence d'externalités sociales positives jusqu'à un certain niveau de fréquentation collective (les pêcheurs aiment à se retrouver entre eux), au-delà duquel les effets négatifs liés aux stocks ou à l'encombrement des parcours l'emportent sur l'agrément social. L'effet positif de la congestion peut être également un artefact économétrique, dans la mesure où les efforts collectifs les plus importants sont souvent observés sur les rivières les plus riches en ressource, ce qui crée de la colinéarité. Les biologistes canadiens COX et WALTERS (2002) ont développé une approche différente sur un échantillon de lacs à truite de Colombie britannique au Canada. Le consentement à payer individuel est indexé positivement sur les captures par unité d'effort (CPUE), plutôt que sur l'effort total. Ils montrent par ailleurs que la CPUE est une fonction décroissante de l'effort total (effet négatif de la congestion).

Dans un deuxième temps, ANDERSON fait dépendre la qualité de la pêche d'améliorations environnementales affectant le stock. Outre la condition évoquée cidessus, l'optimum est alors caractérisé par l'égalité entre le coût marginal de production de l'amélioration environnementale et le gain de bien-être sommé sur toutes les visites, c'est-à-dire le consentement à payer collectif pour bénéficier de cette amélioration. Dans le cas où on a affaire à des biens collectifs qui ne sont pas vendables (situation d'externalité), les agents privés n'entreprennent pas spontanément ces améliorations et la condition d'optimalité n'est pas atteinte. La demande et l'offre potentielle de services environnementaux existent, mais ne se rencontrent pas.

En résumé, l'effort de pêche et la qualité de l'environnement doivent être augmentés jusqu'au niveau où le consentement à payer marginal est égal au coût marginal induit par cette augmentation marginale d'effort ou de qualité du milieu.

Le constat dressé en introduction nous incite à penser que cette gestion optimale de l'effort de pêche et du milieu n'est pas pratiquée en France. Nous allons maintenant montrer ce qui empêche de l'atteindre, à la lumière du fonctionnement des institutions.

\section{L'ANALYSE DE LA GESTION DE LA PÊCHE DE LOISIR EN FRANCE}

L'organisation actuelle de la pêche associative repose sur les lois de 1941 et de 1984 (BRETON, 1993). A partir de 1941, les pêcheurs doivent obligatoirement faire partie d'une association agréée de pêche et de pisciculture (AAPP) et acquitter la taxe piscicole, destinée à financer la surveillance et la mise en valeur du patrimoine piscicole national. La loi de 1984 conserve le cadre associatif et notamment l'obligation d'adhésion. Elle renforce les responsabilités et les moyens des associations, désormais appelées AAPPMA ${ }^{6}$, en matière de protection des milieux aquatiques et de gestion équilibrée des ressources piscicoles. Elle vise également la démocratisation de la pêche et la simplification des règles régissant l'exercice du loisir. Certains auteurs ont parlé de "dilemme associatif " à propos de ces exigences qui peuvent s'avérer contradictoires (AUXIETRE et JANTZEN, 1992).

Les AAPPMA louent ou possèdent les droits de pêche et gèrent les lots correspondants qui sont en principe accessibles à tous. Elles ont l'obligation de s'affilier à des fédérations départementales qui coordonnent leurs actions en matière d'aménagement des milieux aquatiques, en relation avec les préfets et les conseils généraux. Les fédérations départementales peuvent adhérer volontairement à l'Union Nationale pour la Pêche en France et la protection des milieux aquatiques (UNPF). L'UNPF est, avec le Conseil Supérieur de la Pêche (CSP), l'interlocuteur des pouvoirs publics pour la mise

${ }^{6}$ Associations Agréées de Pêche et de Protection des Milieux Aquatiques. 
au point des mesures législatives ou réglementaires, concernant la gestion des milieux aquatiques et l'exercice de la pêche. Elle tente notamment d'harmoniser les conditions de pêche sur l'ensemble du territoire national (voir infra). L'UNPF a été à l'origine de la loi de 1984 et participe activement à la réflexion sur la pêche qui se tient à l'occasion de la réforme de la loi sur l'Eau (UNPF, 2003).

La taxe piscicole disparaîtrait et serait remplacée par une redevance sur l'utilisation de la ressource, prélevée par les agences de l'eau. Le CSP serait dissout et ses agents rejoindraient les services environnementaux déconcentrés de l'État. La loi confirmerait l'obligation d'adhésion à une AAPPMA et créerait une fédération nationale avec adhésion obligatoire des fédérations départementales. La fédération nationale aurait notamment pour mission de gérer en péréquation un fonds spécial dit « de compensation nationale ", alimenté par une « cotisation nationale milieux aquatiques » payée par les pêcheurs.

Le droit de pêche appartient à l'État sur le domaine public ${ }^{7}$ et au propriétaire riverain sur les autres cours d'eau, qualifiés de domaine privé. Le droit de pêche est un droit d'usage accordé en compensation des obligations de curage et d'entretien du lit de la rivière. Cependant, ce droit de propriété ne peut s'exercer pleinement en raison des différentes dispositions législatives ou réglementaires. C'est d'abord l'obligation d'acquitter la taxe piscicole et d'adhérer à une AAPPMA, régulièrement réaffirmée dans la loi, qui s'impose également au propriétaire ou à ses clients, s'il souhaite exploiter sa pêche pour lui-même ou commercialement. Dans ce cas, le propriétaire riverain doit également respecter la réglementation et établir à ses frais un plan de gestion piscicole. C'est ensuite la loi de 1984 qui exige que le propriétaire partage son droit de pêche avec une AAPPMA pour obtenir les aides publiques à l'entretien ${ }^{8}$. Ces dispositions incitent les propriétaires à abandonner l'exercice exclusif des droits de pêche, qui sont le plus souvent partagés gratuitement avec les AAPPMA ${ }^{9}$.

Les AAPPMA délivrent des permis de pêche annuels dont le prix forfaitaire comprend, outre la taxe piscicole, les cotisations statutaires à l'AAPPMA et à la fédération départementale. La taxe piscicole, dont le montant est de $28 €$, finance le Conseil Supérieur de la Pêche et est fixée nationalement. Le montant des cotisations statutaires pour les AAPPMA et fédérations départementales réciprocitaires est très peu variable à l'échelle nationale. Leur montant s'élève respectivement à $20 €$ et $15 €$ en moyenne. Ainsi, une carte de pêche dite "complète " coûte environ $65 €$. Notons qu'il existe un système d'exonération pour les plus jeunes, les invalides et les moins fortunés dont bénéficient $15 \%$ des pêcheurs (CSP, 2003). De plus, certaines pêches sont soumises à des suppléments. C'est le cas, par exemple, pour la pêche des salmonidés migrateurs $(23,50 €)$ ou des civelles $(41 €)$. Le prix total de la "carte de pêche » en France est modique $^{10}$ au regard des tarifs pratiqués à l'étranger et des données de la littérature sur le consentement à payer pour pratiquer la pêche (Tableau $\mathrm{II}^{11}$ ). Des accords de réciprocité existent entre les AAPPMA et entre les fédérations départementales, ce qui permet au pêcheur d'une AAPPMA d'accéder au territoire réciprocitaire en payant seulement une taxe supplémentaire de $16 €$. Sous l'impulsion de I'UNPF, I'Entente Halieutique du Grand Ouest et le Club Halieutique Interdépartemental ${ }^{12}$ ont conclu un accord de réciprocité ouvrant les deux tiers du territoire français (Figure 4).

\footnotetext{
${ }^{7}$ Rivières navigables et flottables.

8 Article L432-1 du Code de l'Environnement.

${ }^{9}$ Avec des exceptions régionales comme en région parisienne ou en Normandie, sur les cours d'eau de première catégorie.

10 Inférieur à $80 €$.

11 Voir AMIGUES, ARNAUD et BONNIEUX (2003) pour un recensement complet des études françaises concernant tous les types de pêche de loisir, y compris en mer.

12 Sud de la France.
} 
Quelques fédérations et AAPPMA, souvent bien pourvues en patrimoine piscicole à forte demande, ont refusé de conclure les accords réciprocitaires (Figure 4). Ceci constitue virtuellement une politique de différentiation tarifaire visant à procurer des ressources financières et limiter la congestion. En outre, certaines d'entre-elles pratiquent des tarifications plus élevées, comme l'Elorn dans le Finistère, la Sée et la Sélune dans la Manche ou encore la Touques dans le Calvados. Quand elle porte sur le prix de la carte annuelle, la différentiation tarifaire affecte le nombre de pêcheurs. A partir de données de panel portant sur les effectifs des pêcheurs de saumon des départements de l'ouest entre 1993 et 2002, SALANIÉ, LE GOFFE et SURRY (2003) montrent qu'une augmentation du supplément migrateurs de $1 €$ diminue la participation de $3 \%$. Seule une différentiation par carte à la journée permettrait de contrôler l'effort individuel.

\section{Tableau II}

Estimations de valeurs journalières de surplus pour la pêche récréative en rivière : études françaises et internationales.

\section{Table II}

Freshwater recreational fishing per day surplus estimates: French and international results.

\begin{tabular}{|c|c|c|c|}
\hline $\begin{array}{l}\text { Études } \\
\text { Studies }\end{array}$ & $\begin{array}{l}\text { Espèces } \\
\text { Species }\end{array}$ & $\begin{array}{l}\text { Régions ou pays } \\
\text { Regions or } \\
\text { countries }\end{array}$ & $\begin{array}{c}\text { Surplus par } \\
\text { jour* } \\
\text { Surplus per } \\
\text { day* }\end{array}$ \\
\hline WALSH et al. (1992) & Poissons anadromes & USA & $62 €$ \\
\hline $\begin{array}{l}\text { BONNIEUX et VERMERSCH } \\
\text { (1993) }\end{array}$ & Saumon & Manche & $51-72 €$ \\
\hline NAVRUD et STRAND (1994) & $\begin{array}{l}\text { Saumon et truite } \\
\text { de mer }\end{array}$ & Norvège & $21-90 €$ \\
\hline \multirow{3}{*}{ AMIGUES et al. (1995) ${ }^{\star \star}$} & \begin{tabular}{|l|} 
Salmonidés \\
sédentaires
\end{tabular} & \multirow{3}{*}{ France } & $19-26 €$ \\
\hline & \begin{tabular}{|l} 
Salmonidés \\
migrateurs
\end{tabular} & & $26-60 €$ \\
\hline & $\begin{array}{l}\text { Cyprinidés et } \\
\text { percidés }\end{array}$ & & $14-22 €$ \\
\hline \multirow{5}{*}{ BOYLE et al. (1998)**** } & Saumon & USA hors Alaska & $25 €$ \\
\hline & \multirow{2}{*}{ Truite } & Ouest des USA & $48 €$ \\
\hline & & Est des USA & $19 €$ \\
\hline & \multirow{2}{*}{$\begin{array}{l}\text { Autres espèces } \\
\text { d'eau douce }\end{array}$} & Ouest des USA & $47 €$ \\
\hline & & Est des USA & $19 €$ \\
\hline STURTEVANT et al. (1998) ${ }^{* * * *}$ & Toutes pêches & Est des USA & $35 €$ \\
\hline \multirow{3}{*}{$\begin{array}{l}\text { ROSENBERGER et LOOMIS } \\
(2001)^{* * *}\end{array}$} & \multirow{3}{*}{ Toutes pêches } & USA (Ensemble) & $22 €$ \\
\hline & & Nord-Est des USA & $16 €$ \\
\hline & & Alaska & $43 €$ \\
\hline MARKOWSKI et al. (2001)**** & Saumon & USA & $62 €$ \\
\hline
\end{tabular}

* Valeurs actualisées pour 2002 à l'aide de l'indice des prix à la consommation de l'INSEE. Les valeurs en dollars ont été converties sur la base de $1 \$=1 €$.

** Valeurs proposées par les auteurs pour la France issues des valeurs étrangères.

*** Ces études sont des analyses statistiques portant sur un grand nombre d'études. La valeur fournie est la médiane de l'échantillon, sauf pour MARKOWSKI et al. et STURTEVANT et al. pour lesquelles nous fournissons la moyenne. 


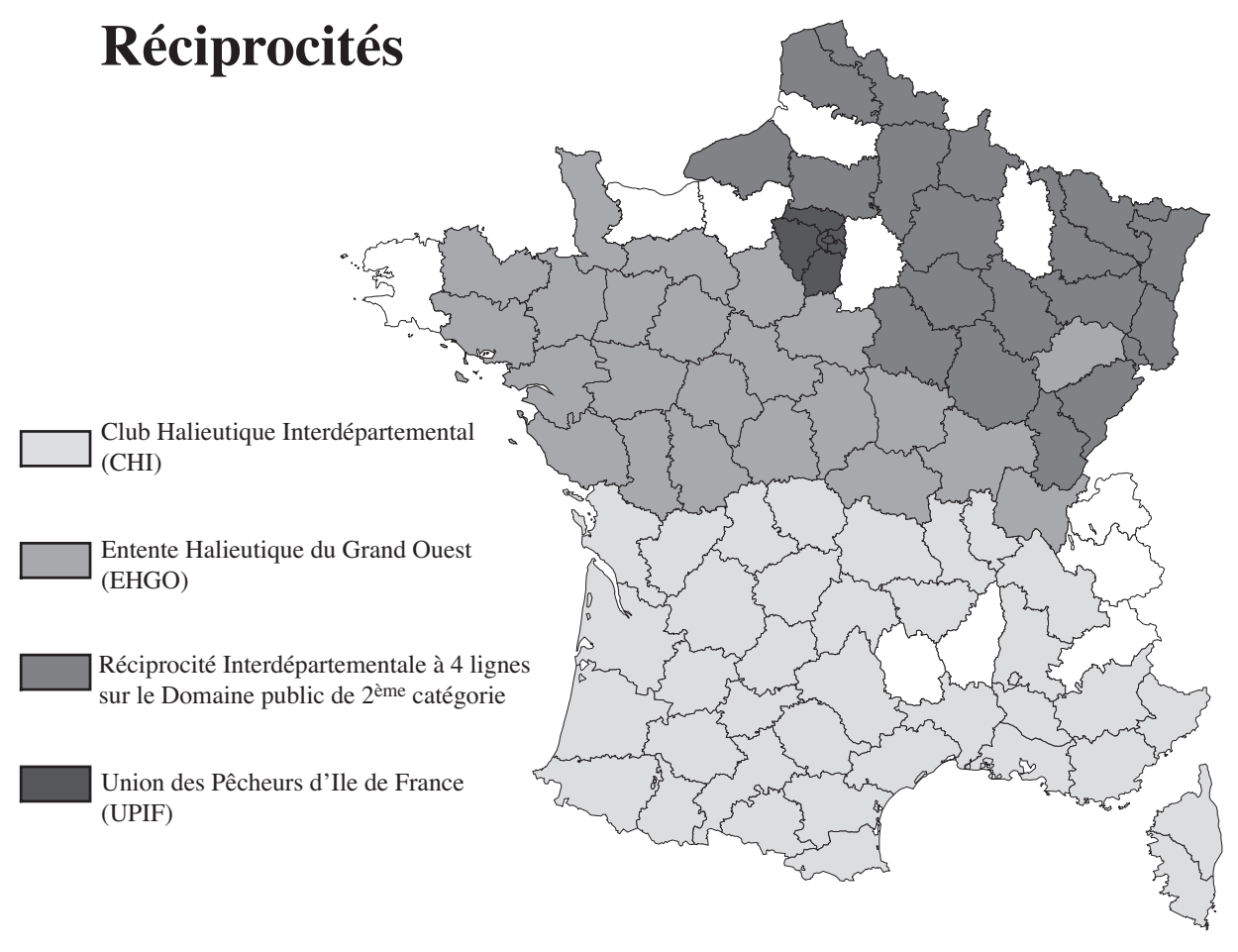

Union Nationale pour la Pêche en France / Janvier 2003

\section{Figure 4}

Carte des réciprocités inter-départementales.

\section{Figure 4 \\ Inter-departemental reciprocities map.}

L'incitation au partage des droits de propriété et la politique des AAPPMA d'accès non contrôlé à un faible prix forfaitaire ont fait passer les droits de propriété du statut de bien privé à celui de bien collectif, avec des possibilités d'exclusion très limitées. Les accords réciprocitaires à grande échelle font que les AAPPMA renoncent également aux droits de propriété qu'elles ont obtenus des riverains, ce qui généralise l'accès non contrôlé. Les externalités qui en résultent empêchent d'atteindre les optima décrits dans la première partie. Les propriétaires riverains ne sont pas incités à fournir des services environnementaux qui ne sont plus commercialisables. L'exemple typique est l'abandon, par les agriculteurs, des prairies de fonds de vallée et l'arrêt de l'entretien des berges, avec des conséquences en terme de détérioration des écosystèmes et de réduction de l'accès aux rivières. De la même façon, les AAPPMA ne sont pas incitées à prendre des mesures d'amélioration et de gestion des ressources piscicoles, puisqu'elles ne peuvent pas en tirer les bénéfices. Ceci constitue une contradiction majeure avec la loi de 1984. Les externalités de stocks sont contestées par les hydrobiologistes, en raison de la faible efficacité de la pêche récréative ${ }^{13}$. Cependant, les externalités de congestion peuvent conduire également à la tragédie des communs. Ce ne sont pas le stock et la rente

\footnotetext{
${ }^{13}$ Sauf pour des ressources menacées, à forte capturabilité par pêche à la ligne, comme le saumon de printemps.
} 
commerciale qui sont affectés comme dans l'exemple de HARDIN (1968), mais la qualité de la pêche et le surplus collectif des pêcheurs.

L'État et les structures de la pêche ont mis en place depuis longtemps des réglementations pour limiter les conséquences de l'accès libre sur les ressources piscicoles. Elles concernent la fermeture de la pêche en période de reproduction, la taille minimale des captures, la limitation journalière des captures et l'interdiction de certains engins ou modes de pêche. Des auteurs ont mis en doute l'intérêt de certaines mesures car il n'y a généralement pas de surexploitation des ressources piscicoles par pêche à la ligne (THIBAULT, 1992). La pêche au saumon fait l'objet de réglementations spécifiques : fermetures hebdomadaires dans certains départements, limitation des captures par rivière et par saison, entraînant la fermeture de la pêche lorsque que le Total Autorisé de Captures (TAC) est atteint. Pour maintenir les stocks, les AAPPMA ont également recours au repeuplement à partir de poissons produits en pisciculture. L'efficacité de cette mesure est également contestées par les hydrobiologistes qui privilégient l'amélioration de la qualité des milieux aquatiques, notamment celles des habitats piscicoles (COWX, 1997).

La plupart de ces mesures s'inspirent plutôt d'une approche biologique de la conservation de la ressource, mais prennent peu en compte le comportement économique du pêcheur ${ }^{14}$. Or, quelle que soit l'efficacité biologique de ces mesures, elles sont insuffisantes du point de vue du bien-être social, car elles s'attaquent aux conséquences de l'accès libre sur la ressource, mais pas à la limitation de l'effort de pêche. COX et WALTERS (2002) ont ainsi montré qu'en accès libre, les mesures destinées à accroître les stocks n'avaient pas d'effet sur la qualité de la pêche, exprimée par les captures par unité d'effort, en raison de l'augmentation de l'effort total avec l'abondance des stocks. Ces observations sont bien expliquées par la théorie économique (tragédie des communs et annulation du consentement à payer marginal). On retrouve ici l'esprit de l'Article L. 430-1 du Code de l'Environnement, qui prône une gestion équilibrée des ressources piscicoles, mais précise également le caractère social et économique de la pêche.

Ne pas intégrer la satisfaction des pêcheurs peut conduire à des effets pervers. Dans le cas du saumon, si la fixation d'un TAC n'est pas contestable, la réduction de la saison de pêche par recul de l'ouverture ou fermeture anticipée peut induire une perte élevée de bien-être, qui pourrait être en partie évitée par une meilleure régulation de l'effort de pêche dans le temps et entre les individus. Parallèlement, la fermeture hebdomadaire peut conduire à un report d'effort sur les journées ouvertes et donc à une baisse de qualité de la pêche due à la congestion supplémentaire, provoquant une perte de bien-être à effort total constant. Ces mesures radicales ont cependant l'avantage d'éviter les coûts de transaction liés au contrôle de l'effort de pêche ${ }^{15}$.

\section{LES PISTES POUR RESTAURER L'OPTIMUM}

Comment prendre en compte les effets externes pour augmenter le bien-être des pêcheurs ? On peut essayer de répondre à cette question dans le cadre coasien des coûts de transaction (COASE, 1960). II s'agit de tous les coûts associés aux opérations nécessaires à la réalisation d'une transaction marchande : prospection, information des agents, négociation, établissement du contrat, exclusion, contrôle et application du contrat, etc. Ces coûts de transaction empêchent le marché de fonctionner quand ils sont

\footnotetext{
${ }^{14} \mathrm{~A}$ propos des mesures à prendre pour développer le tourisme pêche, TENDRON (1996) souligne : "Elles visent à faire en sorte que des dérogations à la réglementation générale ne soient autorisées que pour des impératifs biologiques ».

${ }^{15}$ La congestion des parcours obtenue par la concentration des pêcheurs dans le temps peut être vue comme un moyen peu coûteux de limiter la pression sur la ressource.
} 
supérieurs aux bénéfices espérés des transactions marchandes. La fourniture publique est plus efficace dans ce cas. Les pêcheurs tirent leur satisfaction de la consommation d'un ensemble de biens qui diffèrent par leur caractère plus ou moins collectif ou privé et donc par les coûts de transaction associés à leur fourniture.

On recense d'abord des biens collectifs ayant la caractéristique de ressources communes ou de biens publics, qui nécessitent l'intervention de l'État car ils ne peuvent être gérés par le marché en raison de coûts de transaction importants, notamment ceux touchant à l'exclusion. II s'agit de certaines ressources halieutiques comme les poissons migrateurs (saumon), les ressources en eau, les caractéristiques physiques des bassins versants qui agissent sur le circuit de l'eau, etc. La bonne gestion de ces biens relève des politiques de préservation et de partage des ressources halieutiques, de la politique de l'eau et des politiques agri-environnementales. Ce n'est pas l'objet de l'article que d'examiner ces politiques publiques non spécifiques, c'est pourquoi l'analyse qui suit sera centrée sur les politiques associatives relevant des structures de la pêche.

Deux types de biens sont dans la sphère d'intervention des structures de la pêche. II s'agit d'une part de biens collectifs spécifiques locaux, liés notamment au renouvellement des stocks halieutiques : libre circulation des poissons, zones de frayères, aménagement des milieux, reconstitution des stocks. Pour les mêmes raisons que précédemment, ces biens nécessitent une gestion collective qui sera utilement assurée par les associations et leurs fédérations, comme c'est le cas actuellement : aménagements d'obstacles, mise en réserve et/ou aménagement de frayères, unités de production de juvéniles. Les plans de gestion piscicole, qui sont financés par le CSP, les Agences de l'Eau, les Régions et l'État, constituent un outil déjà opérationnel pour évaluer et restaurer les milieux aquatiques. ARMAND, BONNIEUX ET CHANGEUX (2002) ont étudié la rentabilité de ces plans de gestion en mettant l'accent sur les valeurs associées au poisson sauvage. L'aide de l'État est envisageable ici, compte tenu des valeurs d'usage passif associées à la biodiversité et aux stocks halieutiques.

On a affaire d'autre part à des biens privés gérés en ressources communes, en raison du transfert des droits de propriété des riverains aux AAPPMA : les droits de pêche, l'accès aux berges et les services locaux correspondants, notamment environnementaux. Ces biens sont potentiellement privatisables, car les droits de propriété sont bien définis et les coûts d'exclusion et de transaction sont probablement faibles, puisque la pêche privée en eaux closes se développe. La privatisation des parcours associatifs progresse également, dans les contextes où les biens collectifs non spécifiques sont de bonne qualité (eau et ressource halieutique), malgré les obstacles institutionnels. Bien qu'elle soit un moyen de gérer efficacement l'environnement et les ressources, la privatisation n'est pas souhaitable pour des raisons distributives liées à l'équité dans l'accès. Le défi associatif des prochaines années est d'améliorer l'efficacité économique de la gestion, tout en conservant un accès non discriminatoire. Pour enrayer la privatisation et fournir des services locaux ${ }^{16}$, il faut encourager les AAPPMA à se positionner sur ce marché des droits de pêche, soit en négociant la location et les services avec les riverains, soit en achetant les parcelles. Les riverains, généralement des agriculteurs, sont susceptibles de fournir des services environnementaux à faible coût en raison des économies de gamme ${ }^{17}$. Cette internalisation marchande passe par une augmentation des cotisations.

\footnotetext{
16 On s'en remet aujourd'hui à des politiques publiques comme les mesures agri-environnementales, dont l'efficacité est contestée, notamment en raison de coûts administratifs et de contrôle très élevés. On pourrait envisager que certaines d'entre elles soient gérées par les AAPPMA, pour assurer la transition avec le principe bénéficiaire-payeur.

17 En effet, la production conjointe de biens agricoles et de services environnementaux est souvent moins coûteuse que leur production séparée.
} 
Ce mouvement est encore bien timide et limité aux parcours à forte valorisation (cas des AAPPMA de la Manche qui ont institué un supplément saumon en 2003 pour financer la location des parcours; cas de l'AAPPMA de l'Elorn dans le Finistère qui pratique une politique active d'achat de berges). II conviendrait de rediriger l'argent des pêcheurs vers la négociation locale, ce qui malheureusement ne semble pas être la direction prise par la réforme en cours, qui renforce la centralisation.

Est-il possible que des riverains acquièrent un pouvoir de marché sur le marché des droits de pêche, ce qui serait un obstacle à la maximisation du bien-être collectif, comme le montre la théorie du monopole ${ }^{18}$ ? C'est un phénomène que l'on peut probablement observer en eaux closes où le propriétaire est souvent unique, d'autant plus si la géographie ou des innovations portant sur l'espèce ou la taille des poissons (spécimens) donnent au plan d'eau un caractère unique, au moins localement. Le propriétaire exploitant peut alors avoir intérêt à rationner la demande en fixant des prix élevés pour augmenter son profit. La propriété est beaucoup plus morcelée sur les eaux libres, compte tenu de la longueur des rivières, ce qui limite le risque de pouvoir de marché qui pourrait venir des riverains. En revanche, un particulier a la possibilité de constituer un parcours en achetant des berges ou en louant des droits de pêche. Dans le cas des salmonidés migrateurs, qui concernent peu de rivières en France et des parcours limités souvent concentrés à l'aval des rivières (cas des petites rivières de l'ouest), le risque de monopole existe alors. C'est une raison supplémentaire, au-delà des questions d'équité évoquées plus haut, pour inciter les AAPPMA et leurs fédérations à négocier les droits de pêche ou à acheter des berges. Les cas extrêmes pourraient justifier le recours à l'exercice public d'un droit de préemption, nécessairement limité compte tenu de son coût et des inconvénients de l'aliénation des droits de propriété.

L'autre problème des structures de la pêche, c'est de réguler efficacement et équitablement l'effort de pêche, sans employer les solutions radicales consistant à réduire la période de pêche. Les économistes anglo-saxons s'intéressent depuis longtemps au rationnement des activités récréatives soumises à des coûts de congestion, en utilisant les instruments classiques que sont les quotas et les prix. CULLEN (1985) a comparé les droits d'entrée et les coûts d'accès aux quotas globaux avec loterie ou file d'attente et aux permis individuels, sous l'angle de l'équité et de l'efficacité économique incluant les coûts administratifs. Un résultat classique, également évoqué par ROSENTHAL, LOOMIS et PETERSON (1984) et formalisé par SIBLY (2001), est que les prix sont plus efficaces que n'importe quel autre système de rationnement, car ils allouent davantage de visites à ceux qui les valorisent le plus. A l'opposé, les systèmes de quota global ou individuel autorisent l'exclusion des usagers à valorisation élevée par les usagers à faible valorisation, ce qui conduit à un surplus collectif inférieur. La figure 5, qui représente la réaction de deux types de pêcheurs à la fixation d'un droit d'entrée, illustre ce résultat. Pour une même réduction d'effort, les quotas individuels égalitaires diminuent le surplus collectif de $c+g+h-e$, par rapport à une carte à la journée de prix p. La différentiation des prix en fonction de la congestion des sites permet en outre de redistribuer l'effort entre les sites de manière optimale. Enfin, l'application du principe bénéficiaire-payeur évite de faire porter la pression fiscale sur les non-utilisateurs et rend tangibles les valeurs récréatives, ce qui facilite les arbitrages avec les autres fonctions des ressources naturelles.

COX et WALTERS (2002) notent que l'opposition aux programmes de limitation de l'effort vient surtout des pêcheurs locaux qui ont un accès facile aux cours d'eau. On voit effectivement à la figure 3 que les quotas (mais pas le prix) pénalisent davantage la

\footnotetext{
${ }^{18}$ On pourrait également se demander dans quelle mesure la pêche associative détient une sorte de « monopole institutionnel » sur la gestion des eaux libres, avec les conséquences que cela suppose en terme de rapport qualité/prix.
} 
Demande individuelle inverse

CAP marginal

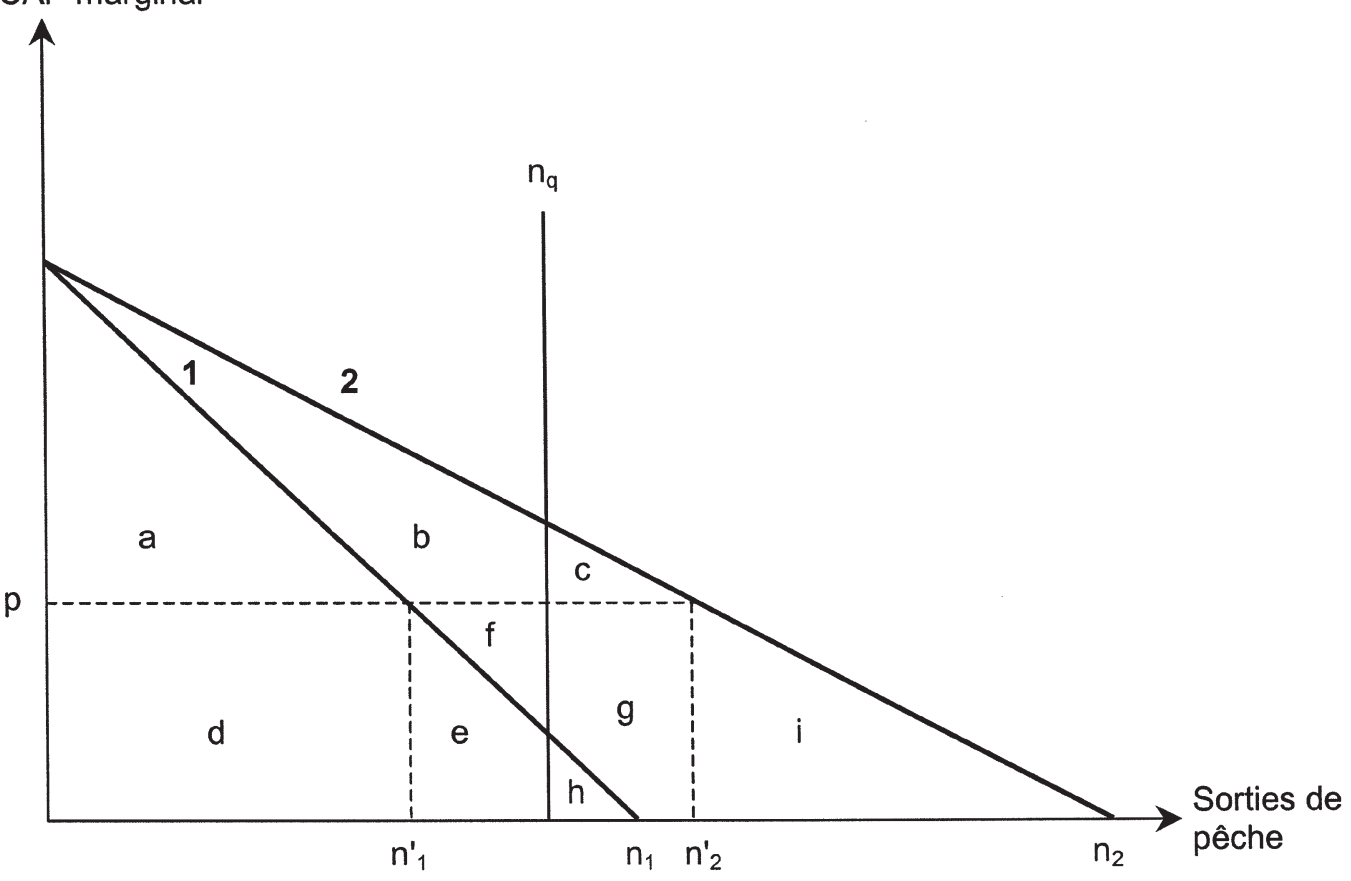

Figure 5

Comparaison d'un quota individuel de jours de pêche $\left(n_{q}\right)$ et d'une carte de pêche à la journée (p) pour deux pêcheurs (1 et 2).

Figure 5

Comparison between an individual fishing days quota $\left(n_{q}\right)$ and a daily entrance fee (p) for two anglers (1 and 2).

fréquentation des pêcheurs qui ont un effort important en l'absence de mesures, ce qui est le cas des pêcheurs locaux. L'accès payant est également considéré comme inéquitable par cette population de pêcheurs ruraux, aux revenus plus modestes que les citadins. Cependant, les différences considérables d'effort individuel observées en accès libre et gratuit ne sont pas plus équitables, dans une vision où il faut participer à la négociation des droits et des services. Par ailleurs, aujourd'hui seuls les pêcheurs riches peuvent accéder à des parcours de qualité, soit en allant à l'étranger soit en louant des parcours privés, ce qui constitue une régulation sauvage et non souhaitée de l'effort. La définition de tarifs sociaux, ou au minimum la différentiation entre locaux et extérieurs, devrait permettre de traiter ces problèmes d'équité. Un système combinant quota forfaitaire de jours, acquis lors de l'achat de la carte de pêche, et paiement à la marge pour les journées supplémentaires (i.e. hors quotas), permettrait également d'inciter à un usage rationnel des ressources, conciliant réduction et redistribution équitable de l'effort.

\section{CONCLUSION}

L'analyse économique et l'examen des quelques données empiriques disponibles en France incitent à se demander si la gestion associative permet de tirer suffisamment de bien-être de la pêche de loisir en eau douce. Notre analyse s'est appuyée sur le cas des salmonidés. D'une part, l'effort de pêche est probablement trop important sur les bons parcours faciles d'accès, ce qui peut entraîner des pertes de bien-être dues à la 
congestion. D'autre part, les propriétaires riverains ne sont pas suffisamment incités à améliorer la qualité de l'environnement, alors que les pêcheurs ont un consentement à payer pour des améliorations. Ces inefficacités s'expliquent par la conjonction de trois phénomènes. Tout d'abord, les droits de propriété, privés, sont atténués par des dispositions législatives. Ensuite, les associations ne contrôlent pas suffisamment l'effort de pêche individuel et collectif. Finalement, les politiques halieutiques sont centrées sur la protection de la ressource et sont, bien que nécessaires, insuffisantes pour maximiser la rente sociale tirée de la pêche.

Nous pensons que si elle veut survivre, ce qui est socialement souhaitable, la pêche associative devrait d'une part mieux reconnaître les droits de propriété en négociant les services environnementaux avec les riverains et d'autre part mettre en œuvre des instruments assurant une régulation efficace et équitable de l'effort de pêche. L'application du principe bénéficiaire-payeur permettrait simultanément de trouver des ressources pour acquérir de véritables droits de pêche et de gérer l'effort individuellement, en allouant les visites à ceux qui les valorisent le plus.

Ces hypothèses demandent à être testées sur des données empiriques. II s'agit, d'une part, de mesurer la demande de pêche récréative, pour en inférer ainsi les coûts de congestion et le consentement à payer pour des améliorations environnementales dans le cas français. D'autre part, il s'agirait également de quantifier les coûts de transaction et de contrôle associés aux diverses options de gestion et les coûts de production des services environnementaux. La mesure de la demande de pêche, et de ses facteurs de variation, devrait permettre d'apporter des éléments tangibles sur certains de ces éléments. La méthode d'évaluation contingente et surtout la méthode des coûts de déplacement devraient être mises en œuvre à partir d'enquêtes du comportement récréatif des pêcheurs. Les modèles multi-sites classiques de la méthode des coûts de déplacement (fonctions de demande de visites estimées à partir de modèles continus et de comptage) et surtout les modèles de choix discret (probabilité de visiter un site) permettraient de relier les comportements récréatifs aux caractéristiques des sites. Des efforts particuliers devraient être faits pour intégrer les problèmes de congestion qui sont capitaux pour la compréhension de la problématique de la pêche de loisir. A notre connaissance, LIN, ADAMS et BERRENS (1996), KERKVLIET et NOWELL (2000), SCHUHMANN et SCHWABE (2004) et SALANIÉ, SURRY et LE GOFFE (2004) constituent les seuls exemples de mesure des externalités de congestion pour la pêche récréative. Les résultats de KERKVLIET et NOWELL et de SCHUHMANN et SCHWABE montrent très clairement l'impact négatif de la congestion sur le bien-être individuel des pêcheurs, au-delà d'un certain niveau d'effort collectif. En outre, ces modèles donneraient des éléments pour identifier les optima d'effort et évaluer les politiques halieutiques comme la réduction de la période de pêche, la limitation de l'effort de pêche par des prix ou des quotas ou la limitation des captures.

II serait également intéressant de comparer la gestion de la pêche en France et à l'étranger. Les systèmes de gestion qui y sont en vigueur sont très différents. Dans les pays du nord de l'Europe, comme la Grande-Bretagne, l'Irlande et la Norvège, la plupart des parcours de pêche sont privés, l'effort de pêche est fortement régulé et l'exercice de la pêche parfois conditionné à l'accompagnement par un guide. En Espagne, le droit de pêche appartient à l'État et plusieurs types de gestion coexistent. Par exemple, les parcours à forte valeur halieutique, les " cotos ", sont régulés par des loteries qui assurent un niveau d'effort faible et l'équité dans l'accès, car les prix à la journée sont maintenus à un bas niveau. II existe par ailleurs des parcours en accès libre, à l'instar de ce qui se fait en France. Aux États-Unis et au Canada, la gestion diffère fortement d'un parcours de pêche à l'autre. Cependant, la plupart des parcours de pêche sont en accès libre. Plusieurs auteurs nord-américains (BERGEN, 2000 ; MINARD, 2000 ; RADOMSKI et al., 2001) soulignent les risques d'une telle gestion, évoquant la possible privatisation des meilleurs parcours de pêche. 
Enfin, le cadre d'analyse développé dans cet article pourrait s'appliquer à d'autres activités de loisirs. C'est particulièrement vrai pour la chasse en France, dont l'organisation est très similaire à celle de la pêche. La randonnée ainsi que la pêche en mer, les sports nautiques ou de montagne, demanderaient un traitement particulier dans la mesure où ils s'appuient sur des biens publics.

\section{RÉFÉRENCES}

AFIT, 1996. La démarche touristique pêche. In : Tourisme-pêche et développement rural, Agence Française de l'Ingénierie Touristique, Paris.

AMIGUES J.P., BONNIEUX F., LE GOFFE P., POINT P., 1995. Valorisation des usages de l'eau, Économica, Paris.

AMIGUES J.P., ARNAUD F., BONNIEUX F., 2003. Évaluation des dommages dans le domaine de l'eau : contribution à la constitution d'une base de données françaises. Rapport INRA, ESR, Rennes, $38 \mathrm{p}$.

ANDERSON L.G., 1983. The demand curve for recreational fishing with an application to stock enhancement activities. Land Economics, 59, 3, 279-286.

ANDERSON L.G., 1993. Toward a complete theory of the utilization and management of recreational fisheries. Journal of Environmental Economics and Management, 24, 272-295.

ANONYME, 1996. Enquête qualitative auprès des clientèles du tourisme halieutique - Résultats de synthèse et premières indications stratégiques. In : Compte rendu des assises nationales du tourisme pêche, Bureau d'audit stratégique CRISTAL, Agence Française de l'Ingénierie Touristique, Paris.

ARMAND C., BONNIEUX F., CHANGEUX T., 2002. Évaluation économique des plans de gestion piscicole. Bulletin Français de la Pêche et de la Pisciculture, 365/366, 565578.

AUXIETRE J.P., JANTZEN J.M., 1992. Les termes du défi associatif, Eaux Libres, 10, 34-35.

BERGEN G., 2000. Limited entry to public trout water the pros and the cons. In: Wild Trout VII - Management in the new millenium: are we ready?, Actes du colloque des 14 octobre 2000, 213-214, Yellow Stone National Park, Montana State University.

BONNIEUX F., VERMERSCH D., 1993. Bénéfices et coûts de la protection de l'eau: application de l'approche contingente à la pêche sportive. Revue d'Économie Politique, 103, 1, 132-152.

BOYLE K.J., BISHOP R.C., CAUDILL J., CHARBONNEAU J., LARSON D., MARKOWSKI M.A., UNSWORTH R.E., PATERSON R.W., 1998. A database of sportfishing values. Economics Division, Fish and Wildlife Service, US Department of the Interior, $77 \mathrm{p}$.

BRETON B., 1993. La pêche en France. Collection Que sais-je ?, $n^{\circ} 2721$, Presses Universitaires de France, Paris.

BRUN A., PINET J.M., 2004. Gestion du cheptel piscicole et surveillance des milieux aquatiques. Le Courrier de l'Environnement de I'INRA, 51, 31-42.

COASE R.H., 1960. The problem of social cost. The Journal of Law and Economics, 3 , $1-44$.

CSP, 1992. La pêche en France : enquête nationale. Eaux Libres, 10, 1-35. 
CSP, 2003. Effectifs réels de pêcheurs par départements en 2003. Conseil Supérieur de la Pêche, Agence Comptable, Paris, 3 p.

COWX I.G., 1997. L'introduction d'espèces étrangères de poissons dans les eaux douces européennes : succès économiques ou désastre écologique ?, Bulletin Français de la Pêche et de la Pisciculture, 344-345, 57-77.

COX S., WALTERS C., 2002. Maintaining quality in recreational fisheries: how success breeds failure in management of open-access sport fisheries, In: PITCHER T.J., HOLLINGWORTH C.E. (Eds.), Recreational Fisheries: Ecological, Economic and Social Evaluation, Blackwell Science, Londres.

CULLEN R., 1985. Rationing recreation use of public land. Journal of Environmental Management, 12, 213-224.

DESAIGUES B., LESGARDS V., LISCIA D., 1999. La valeur de l'eau à usage récréatif : application aux rivières du Limousin, In : POINT P. (Éd.), La valeur économique des hydrosystèmes: méthodes et modèles d'évaluation des services délivrés, Économica, Paris.

FISHER A.C., KRUTILLA J.V., 1972. Determination of optimal capacity of resourced-based recreation facilities. Natural Resources Journal, 12, 417-444.

HARDIN G., 1968. The tragedy of the commons. Science, 162, 1243-1248.

IFEN, 1998. L'environnement en France, Institut Français de l'Environnement. Éditions La Découverte, Paris.

KERKVLIET J., NOWELL C., 2000. Tools for recreation management in parks: the case of the greater Yellowstone's blue-ribbon fishery. Ecological Economics, 34, 89-100.

LAFAGE J.P., 1992. La pêche en France, un potentiel à libérer. Eaux Libres, 10, 19-23.

LIN P.C., ADAMS R.M., BERRENS R.P., 1996. Welfare effects of fishery policies: native american treaty rights and recreational salmon fishing. Journal of Agricultural and Resource Economics, 21, 2, 263-276.

MARKOWSKI M.A., BOYLE K.J., BISHOP R.C., LARSON D.M. et PATERSON R.W., 2001. A cautionary note on interpreting meta analyses. Working Paper, Industrial Economics, Cambridge.

MINARD M., 2000. Limited access fisheries: management options for the future. In: Wild Trout VII - Management in the new millennium: are we ready?, Actes du colloque des 1-4 octobre 2000, 208-210, Yellow Stone National Park, Montana State University.

NAVRUD S., STRAND I., 1994. Norway. In: NAVRUD S. (Ed.), Princing the European Environment, 108-135, Scandinavian University Press, Oslo.

RADOMSKI P.J., GRANT G.C., JACOBSON P.C., COOK M.F., 2001. Visions for recreational fishing regulations. Fisheries, 26, 5, 7-18.

ROSENBERGER R.S., LOOMIS J.B., 2001. Benefit transfer of outdoor recreation use values : A technical document supporting the Forest Service Strategic Plan (2000 revision). General Technical Report RMRS-GTR-72, U.S. Department of Agriculture, Forest Service, Rocky Mountain Research Station.

ROSENTHAL D.H., LOOMIS J.B., PETERSON G.L., 1984. Pricing for efficiency and revenue in public recreation areas. Journal of Leisure Research, 16, 3, 195-208.

SALANIÉ J., LE GOFFE P., SURRY Y., 2003. Évaluation économique des bénéfices récréatifs procurés par le démantèlement des barrages de la Sélune : le cas de la pêche du saumon. Rapport pour la Fédération de la Manche pour la pêche et la protection du milieu aquatique. Agrocampus (DERG), INRA ESR, Rennes, 53 p. 
SALANIÉ J., SURRY Y., LE GOFFE P., 2005. La pêche au saumon dans le massif armoricain : Etat des lieux et mesure de l'effort de pêche, Bull. Fr. Pêche Piscic., $375,35-70$.

SCHUHMANN P.W., SCHWABE K.A., 2004. An analysis of congestion measures and heterogeneous anglers preferences in a random utility model of recreational fishing. Environmental and Resource Economics, 27, 429-450.

SIBLY H., 2001. Pricing and management of recreational activities which use natural resources. Environmental and Resource Economics, 18, 339-354.

STURTEVANT L.A., JOHNSON F.R., DESVOUGES W.H., 1998. A meta-analysis of recreational fishing. Working paper 11-8, Triangle Economic Research, Durham.

TENDRON G., 1996. La démarche touristique pêche. In : Tourisme-pêche et développement rural, Agence Française de l'Ingénierie Touristique, Paris.

THIBAULT M., 1992. Analyse historique de la mise en place des mesures de gestion des ressources halieutiques des eaux continentales. In : OMBREDANE D. (Éd.) Rencontres Halieutiques de Rennes - Les eaux continentales françaises: une richesse à gérer, Actes du Colloque des 28-29 février 1992, Rennes, Agrocampus.

UNPF, 2003. Avenir de la Pêche en France (Texte d'orientation). In : Congrès national des présidents de fédérations de pêche et de protection des milieux aquatiques, Union Nationale pour la Pêche en France, Paris.

WALSH R.G., JOHNSON D.M., McKEAN J.R., 1992. Benefit transfer for outdoor recreation demand studies, 1968-1988. Water Resources Research, 28, 3, 707-713. 